\title{
Dietary patterns, fat and breast cancer recurrence in a UK breast cancer population
}

\author{
S. F. Brennan ${ }^{1}$, J. V. Woodside ${ }^{1}$, M. Lentjes ${ }^{2}$, L. S. Velentzis ${ }^{3}$, K. A. Perkins ${ }^{4}$, A. J. Leathem ${ }^{4}$, \\ M. V. Dwek ${ }^{4}$, R. Swann ${ }^{4}$ and M. M. Cantwell ${ }^{1}$ \\ ${ }^{1}$ Centre for Public Health, Queen's University Belfast, Institute of Clinical Sciences B, Grosvenor Road, \\ Royal Victoria Hospital, Belfast, BT12 6BJ, UK, ${ }^{2}$ Strangeways Research Laboratories, Cambridge, CB1 8RN, UK, \\ ${ }^{3}$ Cancer Epidemiology Research Unit, Cancer Council New South Wales, NSW 1340, Sydney, Australia and \\ ${ }^{4}$ Against Breast Cancer Research Unit, University of Westminster, London, W1W 6UW, UK
}

To date, research into the role of dietary patterns and diet quality on breast cancer prognosis is limited and has mainly been conducted in the United States ${ }^{(1-2)}$, whilst inconsistent results have been observed for dietary fat intake ${ }^{(3-4)}$. As a result, no specific dietary guidelines for cancer survivors are currently available ${ }^{(5)}$. Using information obtained from the UK breast cancer survivors' cohort, the DietCompLyf study, the aim of the present study to examine the relationship between dietary patterns and fat intake and breast cancer recurrence using a nested case-control design.

Cases were participants who had a breast cancer recurrence during the 4-5 year follow up period after diagnosis and these 105 cases were individually matched to one control i.e. women who did not have a recurrence. Dietary data was collected using 7-day food diaries, completed 2 years post-diagnosis, and was entered into DINERWIN/DINERMO. Food groups were formed and factor analysis was used to derive dietary patterns amongst controls. Nutrient analysis included average daily intake $(\mathrm{g})$ of total, polyunsaturated, monounsaturated, saturated and trans fat. Cox proportional hazards regression models were used to determine the association between dietary patterns and fat intake and breast cancer recurrence. Analyses were adjusted for body mass index $\left(\mathrm{Kg} / \mathrm{M}^{2}\right)$, oestrogen receptor status, energy intake (kcals) and post-diagnosis physical activity (MET-h week ${ }^{-1}$ ).

Prudent, High-Energy, Western and Refined dietary patterns were identified from the dietary data of controls. In multivariate adjusted analyses, an increased risk of breast cancer recurrence was observed in women in the middle and highest tertile of consumption of the High-Energy dietary pattern (HR: 3.06; $95 \%$ CI: 1.22, 7.45; HR: $2.71 ; 95 \%$ CI: $1.17,6.28$, respectively; $P_{\text {for trend }}=0.03$ ) compared with women in the lowest tertile. Other dietary patterns (i.e. Prudent, Western and Refined) were not associated with breast cancer recurrence. There was no association between fat intake and breast cancer recurrence.

The results indicate that a High-Energy dietary pattern which included a high intake of high-energy drinks, wine, dressings, potatoes, processed fat, organ meat and high fat dairy increased risk of breast cancer recurrence in women with a previous invasive breast cancer diagnosis. It will be of interest to re-examine these associations when the DietCompLyf cohort has been followed up for a longer period of time and when more breast cancer recurrences have occurred.

1. Kroenke CH, Fung TT, Hu FB \& Holmes MD (2005a) J Clin Oncol 23, 9295-9303.

2. Kwan ML, Weltzien E, Kushi LH et al. (2009) J Clin Oncol, 27, 919-926.

3. Chlebowski RT, Blackburn GL, Thomson CA et al. (2006) J Natl Cancer Inst, 98(24): 1767-1776.

4. Pierce JP, Natarajan L, Caan BJ et al. (2007b) J Am Med Assoc, 298(3): 289-298.

5. World Cancer Research Fund \& American Institute for Cancer Research (2007) Food, Nutrition and the prevention of cancer: a global perspective, $2^{\text {nd }}$ ed. Washington, DC. 